\title{
Efecto del aprendizaje basado en retos sobre las tasas académicas en el área de comunicación de la Universidad Europea de Madrid
}

\author{
Luis A. López-Fraile" María M. Agüero y Eva Jiménez-García ${ }^{2 *}$ \\ (1) Facultad de Ciencias Sociales y de la Comunicación, Dpto. de Comunicación y Marketing. Universidad Europea de \\ Madrid, C. Tajo s/n, Villaviciosa de Odón, 28670, Madrid, España. (Correo-e: luis.lopez@universidadeuropea.es; \\ mariamercedes.aguero@universidadeuropea.es) \\ (2) Vicerrectorado de Profesorado e Investigación. Unidad de Modelo Académico y Transformación Digital, Universidad \\ Europea de Madrid, C. Tajo s/n, Villaviciosa de Odón, 28670, Madrid, España. \\ (Correo-e: eva.jimenez@universidadeuropea.es)
}

* Autora a quien debe ser dirigida la correspondencia

Recibido Feb. 4, 2021; Aceptado Abr. 5, 2021; Versión final May. 28, 2021, Publicado Oct. 2021

\begin{abstract}
Resumen
El objetivo de este estudio es comprobar si la metodología de aprendizaje basada en retos, utilizada en distintos grados del área de comunicación de la Universidad Europea de Madrid, mejora significativamente las tasas académicas de referencia de los programas formativos. El tipo de muestreo es censal, utilizando para ello un estudio de cohorte dinámica retrospectiva donde se comparan dos cohortes: 1) alumnos que utilizaron la metodología y 2) alumnos que no la utilizaron. Por medio de tablas de clasificación cruzada y el estadístico Chi-cuadrado, se contrasta si la utilización de la metodología basada en retos provoca diferencias estadísticamente significativas en las tasas académicas. Los resultados muestran tasas de evaluación, éxito y rendimiento más elevadas, logrando incrementos estadísticamente significativos de entre un $2 \%$ y un $23 \%$ en el grupo de estudiantes que utilizaron la metodología. Se concluye que la metodología de aprendizaje basado en retos tiene un efecto positivo sobre los estudiantes ya que contribuye significativamente a mejorar sus tasas académicas.
\end{abstract}

\section{Effect of challenge-based learning on academic performance rates in communication degree programs at the European University of Madrid}

\begin{abstract}
The main objective of the present study is to assess whether the challenge-based learning methodology, applied in communication degree programs at the European University of Madrid, significantly improves academic reference rates of training programs. The sampling type is a census, using a retrospective dynamic cohort approach where two cohorts are compared: 1) students who used the methodology and 2) students who did not. By means of cross-classification tables and the Chi-square statistic, tests are performed to assess whether the use of the challenge-based methodology causes statistically significant differences in academic rates. The results show high evaluation, success, and performance rates. There are statistically significant increases $(2 \%$ to $23 \%)$ in the student group that used the methodology. It is concluded that the challengebased learning methodology has a positive effect on students since it contributes significantly to improving their academic rates.
\end{abstract}




\section{INTRODUCCIÓN}

Uno de los grandes retos a los que se enfrenta la educación superior y, por ende, uno de los grandes objetivos a conseguir por los profesionales de la enseñanza universitaria es desdibujar las fronteras existentes entre la formación y la profesión, entre lo académico y lo laboral y, por tanto, disminuir la brecha que separa a los egresados universitarios de las necesidades de sus empleadores. Los estudiantes no solo necesitan aprender los conocimientos que los lleven a tener éxito en el mundo laboral; también necesitan desarrollar las competencias que los preparen para un mundo cada vez más globalizado y competitivo. Los estudiantes no solo necesitan sobresalir en áreas como las matemáticas, el lenguaje y la ciencia; también tienen que desarrollar habilidades transversales como el pensamiento crítico, la resolución de problemas, la persistencia y el trabajo colaborativo (Epstein y Hundert, 2002).

El aprendizaje basado en retos (de ahora en adelante ABR), denominado en el ámbito anglosajón challengebased learning al ser creado por la compañía norteamericana Apple en el año 2008 (Yang et al., 2018), es una metodología educativa fundamentada en un soporte experiencial que permite mejorar las competencias de los estudiantes universitarios y adaptar la docencia a los requerimientos del Espacio Europeo de Educación Superior. Esta metodología se define como una pedagogía innovadora caracterizada por la involucración de los estudiantes en contextos y problemas reales y relevantes a los que tienen que dar solución (Lozano et al., 2019). Se diluyen los roles de profesor y alumno, e incluso se incorporan profesionales externos en el proceso, creando equipos de trabajo que desarrollan acciones mancomunadas, consiguiendo así una mayor integración del estudiante con el mundo profesional (Mandeville et al., 2017), lo que permite afrontar con garantías los retos que plantea Bolonia para la educación superior europea. Esta metodología pretende, con desafíos y retos reales, mejorar la formación de los estudiantes y lograr la adquisición de los resultados de aprendizaje previstos en sus titulaciones (Agüero et al., 2019), así como desarrollar competencias como el trabajo en equipo multidisciplinar, la toma de decisiones, el liderazgo y la comunicación (Kohn et al., 2020).

El ABR tiene su origen en el aprendizaje experiencial, bajo el fundamento de que los estudiantes aprenden mejor en situaciones próximas a la realidad, en las que experimentan situaciones a caballo entre el éxito y el fracaso, asumen riesgos y se enfrentan a la incertidumbre. Se trata de un enfoque pedagógico que "aprovecha los intereses de los estudiantes para dar un sentido práctico a la educación" (Portuguez y Gomez, 2020, p.2). Esta metodología se enmarca en el denominado aprendizaje experiencial, enfoque holístico e integrado del aprendizaje que combina la experiencia, la cognición y el comportamiento (Erice et al., 2016). En el caso del ABR, los estudiantes se enfrentan a desafíos reales, dirigidos y dinamizados no sólo por sus profesores, sino también por profesionales de empresas colaboradoras que se involucran en los procesos de enseñanzaaprendizaje, participando de esta manera en el importante rol que la sociedad confiere a los empleadores como actores involucrados en la mejora de las capacidades de los estudiantes, futuros profesionales, para lograr en ellos la adquisición de las competencias que luego les serán vitales en su desempeño laboral (Agüero et al., 2019).

Algunos casos de éxito en la implementación y desarrollo de esta metodología en el ámbito universitario son las Ideas Global Challenge (del MIT), basado en un concurso anual donde los estudiantes aplican sus conocimientos a situaciones del mundo real para abordar problemas relacionados con la calidad de vida de personas de todo el mundo (Agüero et al., 2019). Otro caso, es el el programa internacional Problem Based Learning Model de la Aalborg University donde "los estudiantes realizaron un trabajo de campo de 12 días fuera de la universidad, en concreto en Cuba a principios de marzo de 2017; la idea era resolver tareas reales relacionadas con el desarrollo de proyectos de turismo sostenible" (Clausen y Andersson, 2019, p.2). Por otro lado, el Experiential Learning Collaborative de la Universidad de Connecticut, que utilizan el aprendizaje experiencial como parte fundamental de sus cursos de salud, brindando oportunidades para que los estudiantes conecten su aprendizaje con experiencias externas, lo que les permite analizar, evaluar y documentar experiencias externas, mostrando los resultados puntuaciones más altas de los estudiantes en análisis y razonamiento (Gómez et al., 2018). Por último, el President's Challenge de la Universidad de Harvard, con un programa para estudiantes y exalumnos selectos que no solo afrontan el desafío de comprender mejor las necesidades individuales y de la comunidad, sino que también se convierten en líderes aportando soluciones a esas necesidades (Agüero et al., 2019). Como vemos, las implementaciones de la metodología de ABR se ubican por todo el mundo, tanto en el continente europeo (caso danés de la Aalborg University), como en Estados unidos (en Massachusetts y Connecticut). Todos estos casos se realizan en un contexto universitario, pero las titulaciones a pesar de ser heterogéneas, en todas prevalece el espíritu del modelo de ABR, por tanto, abordan problemas reales de empresas, áreas económicas, y, en definitiva, problemas generales de la sociedad en global y por tanto extrapolables al contexto de nuestro estudio.

Vistos los resultados satisfactorios de estos programas, la Facultad de Ciencias Sociales y de la Comunicación de la Universidad Europea decidió que, a partir del curso 2017-2018, se utilizaría el ABR como metodología educativa que asegurase que los estudiantes se enfrentaran a retos profesionales reales para que la brecha entre formación y profesión fuera lo más reducida posible. A pesar de mencionar las cuatro 
áreas de la facultad, este estudio se centra exclusivamente en el área de Comunicación que agrupa las titulaciones afines, de tal forma que nos permita acotar y abordar las implementaciones relativas a la misma.

\section{OTROS ANTECEDENTES}

Como primer paso para la implementación de la metodología en cada una de las áreas, se decidió acuñar una definición propia: "Metodología de aprendizaje experiencial característica de la Facultad de Ciencias Sociales y de la Comunicación de la Universidad Europea, en la que los estudiantes, de forma colaborativa con sus profesores, se enfrentan a retos reales de organizaciones planteando soluciones realizables". Esta definición se recogió en los documentos de trabajo internos de la Facultad y a partir de ahí todos los profesores empezaron a implementar la metodología en las aulas y a profundizar en sus investigaciones sobre ella, tal y como se recoge en el libro del II Congreso Internacional CBL: "Challenge-Based Learning: un puente metodológico entre la educación superior y el mundo profesional (Pinto y Soto, 2021).

Para seguir con la implantación de la metodología y una vez diseñada la definición y la elección de los elementos diferenciales, tuvieron que delimitarse las «big ideas». Esta decisión fue fundamental porque no solo marca el inicio del proceso de aprendizaje sino también por ser un elemento imprescindible de involucración de estudiantes, profesores y profesionales. La premisa de la que se partió es que fueran temas relacionados con la profesión del área de Comunicación, lo suficientemente amplios y versátiles como para ser sostenibles en el tiempo y que ayudaran a los estudiantes a ser el centro de su propio proceso de aprendizaje y a tener un espíritu crítico, algo que les permitirá llegar a ser los futuros líderes del cambio que necesita la sociedad de hoy en día. Por consenso, los temas que se eligieron como «big ideas» en el área de Comunicación fueron la profesionalización, la diversidad, la innovación, la libertad de expresión, la solidaridad, el espíritu crítico y el emprendimiento.

Las «big ideas» seleccionadas tienen que iniciar el proceso de aprendizaje marcado por la metodología de ABR en los títulos del área de Comunicación, aunque hay que señalar que, en estos programas formativos, la aplicación de la metodología no ha supuesto una revolución, sino más bien una evolución natural de un proceso de adaptación de la enseñanza universitaria a las necesidades del mercado laboral, dado que estos programas formativos son pioneros, dentro de la Facultad, por usar la metodología del aprendizaje experiencial. Desde los inicios del Espacio Europeo de Educación Superior, los Grados del área de Comunicación de la Universidad Europea utilizaron los laboratorios profesionales (televisión, radio, periódico y agencia de publicidad) como ejes vehiculares de la formación con el objetivo de acercar la profesión a los estudiantes y maximizar sus competencias profesionales. La implantación de la metodología de ABR pasa por una serie de fases en el proceso de aprendizaje que, según Portuguez y Gomez (2020), deben incluir los siguientes pasos, representados gráficamente en la figura 1, y que contemplan: las «big ideas» (puntos clave o parámetros centrales sobre los que girarán los desafíos), desarrollar las cuestiones esenciales, los retos, actividades y cuestiones guía, generar soluciones y publicar el proceso. El esquema de la metodología de ABR presentado atiende al modelo CANVAS que permite documentar todo el proceso de ABR, así como el análisis y la reflexión, facilitando la retroalimentación con los estudiantes, haciendo así que el proceso sea realmente rico y relevante para ellos.

\begin{tabular}{|c|c|c|}
\hline & a: ¿Qué concepto/idea inspira e & \\
\hline Pre & tta Esencial: ¿Qué problema ten & \\
\hline EIF & : Qué reto planteamos? j para & \\
\hline $\begin{array}{l}\text { Preguntas Guía: } \\
\text { ¿Qué preguntas guiarán el reto a } \\
\text { alcanzar por los estudiantes? }\end{array}$ & $\begin{array}{l}\text { Actividades Guía: } \\
\text { ¿Qué tenemos que hacer para } \\
\text { alcanzar el reto final? }\end{array}$ & $\begin{array}{l}\text { Recursos Guía: } \\
\text { ¿Qué documentos/apps/webs o } \\
\text { herramientas TICS necesitamos? }\end{array}$ \\
\hline Compet & ias Clave: ¿Qué competencias c & se desarrollan? \\
\hline Cont & o: ¿Qué contenido teórico se vir & con el reto? \\
\hline Organización: ¿Cómo se & a agrupar el alumnado y organi & actividad si es multidisciplinar? \\
\hline Plan De Evaluación & La Solución: ¿Qué herramientas & valuación vamos a aplicar? \\
\hline & ón: ¿Cómo vamos a difundir nue & oroyecto? \\
\hline
\end{tabular}

Fig. 1: Esquema del modelo de ABR utilizando CANVAS 
Conocidas las "big ideas", es el momento de identificar los retos. En este sentido, nos parece relevante destacar, a modo de ejemplo altamente significativo, los dos retos que se desarrollaron con importantes empresas que actuaron como partners estratégicos, buscando el objetivo de formar a los alumnos en los contenidos y las competencias más cercanas a la profesión. Se tratan de los desafíos con Comunica $+\mathrm{A}$ y con Telepizza. 1) Reto con Comunica + A: esta importante agencia de publicidad española busca, de manera continua, atraer a jóvenes talentos. La manera de encontrar ese talento y de reducir su periodo de formación interno y su integración en la empresa es a través de su implicación, como partner, en la metodología de ABR de la Facultad. Para ello, cada año lanza un reto a los estudiantes de los grados del área de comunicación de la Facultad. Este reto es real, y está propuesto por uno de los clientes de la agencia, y busca dar solución a alguno de sus problemas de comunicación. Como resultado del reto, y si la evaluación realizada es satisfactoria, el grupo de estudiantes que mejor hayan resuelto el desafío, realizan unas prácticas profesionales remuneradas en la agencia; 2) Reto Telepizza: el grupo Telepizza (grupo de restauración rápida) lanza anualmente un reto a distintas universidades españolas y latinoamericanas (grados del área de comunicación) con el objetivo de identificar jóvenes talentos universitarios de cara a formarles para el mundo real. Telepizza pasa a ser partner de la universidad y plantea un desafío distinto en cada una de las ediciones. En la última edición, el briefing sobre el que trabajaron los estudiantes consistió en plantear ideas para hacer crecer la colaboración entre Telepizza y LaLiga. Como resultado del reto y en función de la evaluación, los alumnos con mejor puntuación tendrán la oportunidad de participar en un programa de formación exclusivo impartido por LaLiga Business School.

En estos dos retos trabajan alumnos de los grados mencionados en este estudio, buscando las sinergias adecuadas, algo muy habitual en la realidad profesional, para vehicular el reto y alcanzar los resultados de aprendizaje establecidos. Para la evaluación de los grupos se trabaja de manera conjunta entre profesores y profesionales de estas empresas y se utilizan las rúbricas apropiadas para la medición de competencias y conocimientos específicos de las titulaciones y, además, se utilizan las rúbricas específicas para evaluar la metodología de ABR. Una vez realizados los retos y para abundar en el proceso de medición y posterior divulgación de los resultados, se realizaron estudios de percepción a los alumnos participantes en el reto. Cabe resaltar que el índice de percepción mayor es en el área de empleabilidad (con un 4 sobre 5) (Agüero et al, 2019), lo que ratifica la elección de la "big idea" profesionalización como hilo conductor del ABR. Además, esta metodología se implementaría gracias a unos elementos diferenciales de los que ya disponía la facultad, concretamente para el área de comunicación se cuenta con Europeamedia - laboratorio de comunicación con vinculación de partners profesionales (convenios formativos con más de 100 empresas, instituciones y ONGs), implicación del claustro (habiendo recibido talleres de formación), desarrollo de competencias cruciales para el mundo profesional, estudiantes como protagonistas de su propio aprendizaje, transferencia de conocimiento universidad-empresa, evaluación externa y, por último, desarrollo del talento individual y colectivo.

\section{TASAS ACADÉMICAS}

El Parlamento Europeo y el Consejo de la UE promulgaron la Recomendación 2006/143/CE, que instaba a todas las instituciones de educación superior a seguir unos estándares y unas directrices que aseguren la calidad en los programas formativos, y plantearon sus requisitos en cuestión de información y rendimiento de cuentas a la sociedad (apartados 1.6 y 1.7). La información y la recogida de datos promoverá la capacidad autocrítica de las instituciones y ayudará a implementar aquellos procesos de mejora continua que la sociedad merece. En este sentido, el Real Decreto 1393/2007, de 29 de octubre, establece, por un lado, la autonomía universitaria de modo que, a partir de su promulgación, son las universidades las que crean y proponen las enseñanzas y programas que imparten. De esta manera, permite la flexibilización y la diversidad de los currículums, cediendo a las universidades la capacidad para la innovación y la creación de títulos que den respuesta a las demandas de la sociedad. Pero también, por otro lado, recoge la necesidad de impulsar y coordinar la creación de un Sistema Integrado de Información Universitaria que dé cobertura a las necesidades de información del conjunto del sistema universitario español, que garantice la homogeneización en la obtención de los datos, que permita la comparación y que garantice la fiabilidad tanto en sus definiciones y metodologías como en los procesos de validación aplicados.

Entre los procesos de garantía en la información que establecen el Real Decreto y la Recomendación citados están los sistemas de verificación, seguimiento y acreditación que debe pasar cada título universitario. Desde los principios de la creación y el diseño del título, deben contemplarse los siguientes elementos: justificación, objetivos, admisión de estudiantes, contenidos, planificación, recursos, resultados previstos y sistema de garantía de calidad. Es en el apartado de resultados previstos en el que cada una de las universidades debe contemplar los indicadores óptimos de eficacia del título, teniendo en cuenta el perfil de ingreso de los estudiantes, los objetivos planteados o el tiempo de dedicación a las asignaturas. Dicho de otro modo, las tasas del título. Entre las tasas que suelen recoger las universidades en relación con los títulos están la tasa de abandono, la tasa de graduación, la tasa de eficiencia, la tasa de evaluación, la tasa de éxito y la tasa de 
rendimiento (García, 2015). Para comprobar la eficacia en la implantación de la metodología de ABR hemos seleccionado las tasas de evaluación, rendimiento y éxito. El motivo de dicha selección es porque los créditos superados (notas) son los indicadores comúnmente utilizados para medir y cuantificar el rendimiento académico (Soto y Amores, 2020). Además de ser un factor fundamental para conocer la calidad educativa, este rendimiento académico, medido por el número de créditos aprobados frente a los créditos matriculados y/o presentados, dan como resultado las tasas objeto de estudio de este trabajo que permiten "conocer la relación entre lo que se aprende y lo que se logra desde el punto de vista del aprendizaje" (Garbanzo, 2007, p.61).

Las definiciones de tasa de evaluación, rendimiento y éxito, atendiendo a Martín et al., (2008), son: 1) Tasa de evaluación: es la relación porcentual entre el número de créditos a los que el estudiante se presenta en un curso académico y el número de créditos que dicho estudiante ha matriculado. Para entenderlo de forma más sencilla, es la tasa de estudiantes que se presentan a examen; 2) Tasa de rendimiento: es la relación porcentual entre el número de créditos superados en un curso académico y el número de créditos matriculados. Es decir, la tasa de aprobados; 3) Tasa de éxito: es la relación porcentual entre el número de créditos superados en un curso académico y el número de créditos presentados. Es decir, la tasa de aprobados sobre los presentados a examen. Para la estimación de estas tasas se utilizan los créditos ECTS (Sistema Europeo de Transferencia de Créditos) enmarcados dentro del Espacio Europeo de Educación Superior. El crédito ECTS es utilizado como unidad de medida de la dedicación que requieren, para un estudiante, un determinado programa, titulación o asignatura. Un crédito tiene una equivalencia entre 25 y 30 horas de trabajo (Comisión Europea, 2017).

\section{METODOLOGÍA}

Conocidos los antecedentes, a continuación, se presenta la metodología utilizada en este estudio, detallando los objetivos de la investigación, el diseño metodológico y muestral, así como los instrumentos para la recogida de datos.

\section{Objetivos}

El propósito fundamental del trabajo presentado es comprobar si la metodología de aprendizaje basada en retos, utilizada en distintos Grados del área de Comunicación de la Universidad Europea de Madrid mejora significativamente las tasas académicas de referencia de los programas formativos. Por tanto, el presente estudio indaga sobre la siguiente hipótesis de partida: la implementación de la metodología de ABR mejora porcentualmente los índices de las tasas académicas de referencia en los títulos (tasa de evaluación, tasa de rendimiento y tasa de éxito).

\section{Diseño metodológico y muestral}

El presente estudio contempla cuatro titulaciones de Grado presenciales del área de Comunicación de la Universidad Europea de Madrid (Grado en Comunicación Audiovisual y Multimedia, Grado en Comunicación Publicitaria, Grado en Marketing y Dirección Comercial y Grado en Periodismo). El motivo por el que se seleccionaron estas titulaciones se debe a que la temática de los desafíos era muy similar entre estas titulaciones. Además, en estas titulaciones contábamos con la experiencia acumulada de dos desafíos de especial relevancia con partners estratégicos externos a la universidad (Comunica+a y Telepizza) de alto nivel en el mundo de la comunicación que se habían desarrollado durante varios cursos académicos y que nos ha parecido relevante destacar.

El procedimiento utilizado se basa en un estudio de cohorte dinámica retrospectiva donde se comparan dos cohortes. La cohorte 1 corresponde con los cursos académicos 2015-2016 y 2016-2017 donde no se aplica la metodología y se conoce como grupo de control. En cuanto a la cohorte 2 corresponde con los cursos 20172018 y 2018-2019, donde sí se aplica la metodología, puesto que desde el curso 2017-2018 fue una metodología que se implantó de forma obligatoria en la Facultad en todas las asignaturas (exceptuando Trabajo fin de Grado y Prácticas) de todos los cursos (1은 $2^{\circ}, 3^{\circ}$ y $4^{\circ}$ ) en las cuatro titulaciones estudiadas. El tipo de muestreo utilizado es censal utilizando para ello cohortes dinámicas dado que los participantes se incluyen a medida que el estudio va avanzando porque en el curso 2015-2016 contamos con estudiantes de $1^{\circ}, 2^{\circ}, 3^{\circ}$ y $4^{\circ}$ de las titulaciones mencionadas, pero de estos cuatro cursos, en el siguiente curso académico 2016-2017 solo participaron los mismos estudiantes de $1^{\circ}, 2^{\circ}$ y $3^{\circ}$ que ahora pasaban a estar en $2^{\circ}, 3^{\circ}$ y y $4^{\circ}$, y en los sucesivos cursos académicos sucede lo mismo y, además, se incluyen los estudiantes de nuevo ingreso. Por lo que, en la última cohorte (2018-2019) de los originales participantes del curso 2015-2016, ya solo quedaban los alumnos de 1으 que ahora pasan a estar en 4ำ curso y todos los estudiantes de nuevo ingreso que se han ido incorporando en los diferentes cursos académicos. Por tanto, el total de estudiantes que participan en el estudio es de 933, distribuidos en los dos grupos, tal y como se muestra en la tabla 1. 
Tabla 1: Descripción de la muestra (Datos de los Registros de la Universidad Europea de Madrid)

\begin{tabular}{|l|c|c|c|c|c|c|}
\cline { 2 - 7 } \multicolumn{1}{c|}{} & \multicolumn{3}{c|}{ Sin ABR } & \multicolumn{3}{c|}{ Con ABR } \\
\cline { 2 - 7 } \multicolumn{1}{c|}{} & $\begin{array}{c}\text { Curso } \\
2015-2016\end{array}$ & $\begin{array}{c}\text { Curso } \\
2016-2017\end{array}$ & Total & $\begin{array}{c}\text { Curso } \\
2017-2018\end{array}$ & $\begin{array}{c}\text { Curso } \\
2018-2019\end{array}$ & Total \\
\hline Grado en Comunicación Audiovisual y Multimedia & 78 & 54 & 131 & 68 & 26 & 93 \\
\hline Grado en Comunicación Publicitaria & 43 & 36 & 79 & 84 & 66 & 150 \\
\hline Grado en Marketing y Dirección Comercial & 95 & 85 & 180 & 68 & 50 & 117 \\
\hline Grado en Periodismo & 29 & 31 & 60 & 63 & 61 & 123 \\
\hline
\end{tabular}

El inicio del estudio es retrospectivo, es decir, posteriori a los hechos estudiados ya que los datos son extraídos una vez que ya se ha aplicado o no la metodología. Para la estimación de las tasas se requiere del número de créditos matriculados, presentados y aprobados que son variables que siempre se registran por curso académico y, por tanto, son fácilmente comparables por grupo (experimental y control). En este sentido y en lo que respecta a la cantidad de créditos y trabajo en cada curso académico, la distribución de los créditos ECTS por curso académico en ambos grupos es de aproximadamente 40 ECTS por curso académico (habiendo eliminado de este cómputo las asignaturas de Trabajo fin de Grado y Prácticas), lo que equivale a una carga de trabajo de entre 1.000 y 1.200 horas.

Hemos de indicar que el resto de las variables más significativas que podrían afectar al proceso de enseñanzaaprendizaje y, por lo tanto, afectar a la fiabilidad del presente trabajo, como son la composición del claustro, las instalaciones, los recursos disponibles y los horarios, han permanecido prácticamente invariables a lo largo de todo el periodo investigado y no han sufrido modificaciones significativas que superen el margen de error estadístico tolerable de un $5 \%$ que hemos mantenido en todo el estudio. Por ejemplo, se ha verificado que la composición del claustro no ha cambiado prácticamente en los cursos que van de 2015 a 2019 , manteniéndose en un 95\% la misma composición y número de profesores. La asignación docente, es decir, el profesor asignado a cada asignatura se ha mantenido también prácticamente invariable en todo el periodo (más de un $96 \%$ de profesores han impartido las mismas asignaturas). También se ha verificado que las instalaciones y recursos han sido los mismos durante todo el periodo, con alguna actualización que no supera el $4 \%$. En cuanto a los horarios, también son esencialmente similares. De esta manera, podemos atribuir los resultados de las tasas académicas a la puesta en marcha de la metodología de ABR.

\section{Recolección y análisis de datos}

Dado que el estudio llevado a cabo se centra en la comparativa de tasas académicas, no se requiere de ningún instrumento, pero sí de los datos provenientes de la información que obra en los registros de la Universidad Europea de Madrid que es utilizada para la estimación de las tasas académicas atendiendo a la definición establecida en el Sistema Integrado de Información Universitaria ([SIIU], 2019).

En lo que respecta al análisis de datos, se ha utilizado una metodología de carácter cuantitativo. Para la toma de decisiones sobre la prueba estadística a utilizar se ha realizado la prueba de contraste KolmogorovSmirnov que es la prueba estadística que permite verificar si los datos de la muestra proceden de una distribución normal (Mishra et al., 2019). Los resultados indican que la significación estadística (sig. asintót. Bilateral) es de 0.000 inferior al nivel de significación establecido para esta investigación $(\alpha=0.05)$, que corresponde con el comúnmente utilizado para el contraste de hipótesis. Por todo ello, se rechaza la hipótesis nula y se concluye que la muestra no sigue una distribución normal y, en consecuencia, se utiliza una prueba no paramétrica mediante tablas de clasificación cruzada entre los grupos y aplicando el estadístico de contraste Chi-cuadrado que es estimado con el programa estadístico SPSS, versión 22.

\section{RESULTADOS}

El apartado de resultados está estructurado en tres bloques, para poder entender de forma más cómoda las derivaciones obtenidas en la investigación. En el primero de ellos se presentan los resultados correspondientes a la tasa de evaluación, en el segundo los resultados de la tasa de éxito y, por último, se muestran los resultados de la tasa de rendimiento en cada una de las titulaciones estudiadas (Grado en Comunicación Audiovisual y Multimedia, Grado en Comunicación Publicitaria, Grado en Marketing y Dirección Comercial y Grado en Periodismo).

\section{Tasa de evaluación}

En la tabla 2 se muestran los resultados de la tasa de evaluación y la diferencia porcentual por titulación. En el caso del Grado en Marketing y Dirección Comercial es la titulación que presenta mayor incremento en la tasa de evaluación. Es decir, la tasa de estudiantes que se presentan a examen aumenta un $11 \%$ en el grupo en el que se utiliza la metodología de ABR frente al grupo que no lo utiliza. También existen mejoras en la 
tasa de evaluación en el resto de las titulaciones, pero con un incremento menor. En lo que concierne al Grado en Comunicación Audiovisual y Multimedia y al Grado en Periodismo, los resultados son similares con incrementos de un $6 \%$ y $5 \%$ respectivamente a favor del grupo que utiliza la metodología. El Grado en Comunicación Publicitaria presenta pequeños incrementos en el grupo que utilizó la metodología frente al grupo que no la utilizó. En concreto, en la tasa de evaluación el incremento es de un $2 \%$.

Para profundizar más en el análisis de los datos extraídos en nuestra investigación de campo, se analizan los incrementos mencionados anteriormente para comprobar si son significativos. En la parte izquierda de la tabla 2 (Prueba Chi-cuadrado), se muestran los resultados del contraste de hipótesis llevado a cabo. En el Grado en Comunicación Audiovisual y Multimedia, el Grado en Periodismo y el Grado en Marketing y Dirección Comercial se rechaza la hipótesis nula dado que la probabilidad asociada al estadístico Chi-cuadrado es inferior al nivel de significación $(\alpha=0.05)$ y se contrasta el efecto positivo que tiene la utilización del ABR en la tasa de estudiantes presentados a examen (tasa de evaluación). Por otro lado, el Grado en Comunicación Publicitaria no muestra un incremento porcentual significativo en la tasa de evaluación en el grupo que utiliza la metodología. No obstante, a pesar de no ser significativo, sí se observa un incremento del $2 \%$ en la tasa, por lo que el efecto de la metodología no deja de ser positivo y favorecedor para el aprendizaje y rendimiento del estudiante.

Tabla 2: Porcentajes y estadísticos de contraste de la tasa de evaluación por titulación

\begin{tabular}{|l|c|c|c|c|c|c|}
\hline & \multicolumn{3}{|c|}{ Porcentajes } & \multicolumn{2}{c|}{ Prueba Chi-cuadrado } \\
\cline { 2 - 6 } & Con ABR & Sin ABR & $\begin{array}{c}\text { Diferencia } \\
\text { porcentual }\end{array}$ & Valor & gl & Sig. \\
\hline Grado en Comunicación Audiovisual y Multimedia & $94 \%$ & $88 \%$ & $6 \%$ & 9.041 & 1 & 0.003 \\
\hline Grado en Comunicación Publicitaria & $95 \%$ & $93 \%$ & $2 \%$ & 1.478 & 1 & 0.233 \\
\hline Grado en Marketing y Dirección Comercial & $96 \%$ & $84 \%$ & $11 \%$ & 40.772 & 1 & 0.000 \\
\hline Grado en Periodismo & $87 \%$ & $82 \%$ & $5 \%$ & 4.845 & 1 & 0.033 \\
\hline
\end{tabular}

\section{Tasa de éxito}

En la tabla 3 se muestran los resultados de la tasa de éxito y la diferencia porcentual por titulación. En el Grado en Marketing y Dirección Comercial, de nuevo es donde se produce el mayor incremento en la tasa de éxito y, por tanto, los alumnos que se presentan a examen logran mayor número de aprobados, en concreto un $14 \%$ más cuando se utiliza la metodología de ABR. Con respecto al Grado en Comunicación Publicitaria, se observa un incremento del $5 \%$ en el grupo que utilizó la metodología frente al grupo que no la utilizó. En el caso del Grado en Comunicación Audiovisual y Multimedia y del Grado en Periodismo, los resultados son similares, con incrementos de un $2 \%$ y $3 \%$ respectivamente, a favor del grupo que utiliza la metodología.

Con la intención de conocer si dichos incrementos son significativos, en la parte izquierda de la tabla 3 (Prueba Chi-cuadrado), se presenta los resultados del contraste de hipótesis. En lo que concierne a la tasa de éxito, en el Grado en Comunicación Publicitaria y en el Grado en Marketing y Dirección Comercial se rechaza la hipótesis nula dado que la probabilidad asociada al estadístico Chi-cuadrado es inferior al nivel de significación $(\alpha=0.05)$ y se contrasta el efecto positivo que tiene la utilización del ABR en la tasa de aprobados sobre los presentados a examen (tasa de éxito). Por otro lado, el Grado en Comunicación Audiovisual y Multimedia y el Grado en Periodismo no muestran un incremento porcentual significativo en la tasa de éxito en el grupo que utiliza la metodología. No obstante, a pesar de que dicha diferencia no sea significativa, se observa que la utilización del ABR provoca un incremento en el número de aprobados sobre los presentados a examen del $2 \%$ en el Grado en Comunicación Audiovisual y Multimedia y un incremento del 3\% en el Grado en Periodismo.

Tabla 3: Porcentajes y estadísticos de contraste de la tasa de éxito por titulación

\begin{tabular}{|l|c|c|c|c|c|c|}
\hline & \multicolumn{3}{|c|}{ Porcentajes } & \multicolumn{3}{|c|}{ Prueba Chi-cuadrado } \\
\cline { 2 - 7 } & Con ABR & Sin ABR & $\begin{array}{c}\text { Diferencia } \\
\text { porcentual }\end{array}$ & Valor & gl & Sig. \\
\hline Grado en Comunicación Audiovisual y Multimedia & $95 \%$ & $93 \%$ & $2 \%$ & 1.319 & 1 & 0.295 \\
\hline Grado en Comunicación Publicitaria & $99 \%$ & $94 \%$ & $5 \%$ & 16.703 & 1 & 0.000 \\
\hline Grado en Marketing y Dirección Comercial & $99 \%$ & $85 \%$ & $14 \%$ & 65.057 & 1 & 0.000 \\
\hline Grado en Periodismo & $93 \%$ & $90 \%$ & $3 \%$ & 2.747 & 1 & 0.118 \\
\hline
\end{tabular}

\section{Tasa de rendimiento}

En la tabla 4 se muestran los resultados de la tasa de rendimiento y la diferencia porcentual por titulación, donde puede verse que el Grado en Marketing y Dirección Comercial de nuevo es la titulación que presenta mayor incremento, en concreto de un $23 \%$ en la tasa de aprobados en el grupo que utiliza la metodología de ABR. Por otro lado, tanto el Grado en Comunicación Audiovisual y Multimedia y el Grado en Periodismo 
presentan el mismo incremento, concretamente de un 7\% para el grupo que utiliza la metodología de ABR. Por último, en el caso del Grado en Comunicación Publicitaria el incremento es del 6\%.

Para corroborar que los incrementos en la tasa de rendimiento son significativos, se realiza en la parte izquierda de la tabla 4 (Prueba Chi-cuadrado) el contraste de hipótesis. Cuyos resultados muestran que esta metodología presenta un efecto positivo y estadísticamente significativo en la tasa de rendimiento de todas las titulaciones (Grado en Comunicación Audiovisual y Multimedia, Grado en Comunicación Publicitaria, Grado en Marketing y Dirección Comercial y Grado en Periodismo). Es decir, se rechaza la hipótesis nula dado que la probabilidad asociada al estadístico Chi-cuadrado es inferior al nivel de significación $(\alpha=0.05)$ y se contrasta el efecto positivo que tiene la utilización del ABR en la tasa de aprobados (tasa de rendimiento).

Tabla 4: Porcentajes y estadísticos de contraste de la tasa de rendimiento por titulación

\begin{tabular}{|l|c|c|c|c|c|c|}
\hline & \multicolumn{3}{|c|}{ Porcentajes } & \multicolumn{2}{c|}{ Prueba Chi-cuadrado } \\
\cline { 2 - 7 } & Con ABR & Sin ABR & $\begin{array}{c}\text { Diferencia } \\
\text { porcentual }\end{array}$ & Valor & gl & Sig. \\
\hline Grado en Comunicación Audiovisual y Multimedia & $89 \%$ & $82 \%$ & $7 \%$ & 9.507 & 1 & 0.002 \\
\hline Grado en Comunicación Publicitaria & $94 \%$ & $88 \%$ & $6 \%$ & 12.285 & 1 & 0.000 \\
\hline Grado en Marketing y Dirección Comercial & $95 \%$ & $72 \%$ & $23 \%$ & 107.777 & 1 & 0.000 \\
\hline Grado en Periodismo & $81 \%$ & $74 \%$ & $7 \%$ & 7.586 & 1 & 0.004 \\
\hline
\end{tabular}

\section{DISCUSIÓN}

El análisis realizado nos muestra que las tasas de evaluación, éxito y rendimiento son superiores en el grupo en el que se ha utilizado la metodología (Con ABR) que en el grupo en el que no se utilizó. Este resultado concuerda con numerosas experiencias realizadas en otros centros universitarios que han tenido el mismo efecto exitoso al aplicar la metodología del ABR. El Observatorio de Innovación Educativa, perteneciente al Instituto Tecnológico y de Estudios Superiores de Monterrey, ha recopilado información sobre numerosos centros universitarios donde el resultado exitoso se ha mantenido (Félix-Herrán et al., 2019; MembrilloHernández et al., 2018). Entre las que destacan la Universidad de Cincinnati, la Universidad Estatal de Montana, la University of Western Australia, el Tecnológico de Georgia o la Chalmers University. Todas las experiencias llevadas a cabo en estas instituciones coinciden en los elementos clave de su implementación: se generan soluciones a problemas reales de índole local o global, donde el aprendizaje difumina los límites entre alumno y profesor, creando procesos de adquisición de habilidades y conocimientos a través del proceso experiencial, que permite desarrollar acciones reales que resuelven verdaderos problemas (Bashir et al., 2017). Las pequeñas diferencias en la implementación estriban en que en algunos casos se afrontan problemas locales, en otras ocasiones son cuestiones de una comunidad humana más amplia y en otras el formato es el de un concurso anual donde abordar problemas de calidad de vida de personas de todo el mundo. La aplicación de la metodología de ABR alcanza ámbitos del conocimiento totalmente ecléctico, incluyéndose áreas de salud, de la ciencia social, de la comunicación, del mundo empresarial o incluso de aspectos intrínsecamente relacionados con la calidad de vida de las personas, por lo que se manifiesta como un proceso aplicable a todas las facultades universitarias.

Este efecto beneficioso también ha sido comprobado, tanto en contextos empresariales (O'Mahony et al., 2012), como en otras áreas de conocimiento, como los de la Pedagogía, la Educación Social o la Educación Infantil, donde otros autores han constatado similares efectos, altamente positivos, comprobando que aumenta el rendimiento, la motivación, y la capacidad de trabajo en grupo del alumnado (Lonka et al., 2004), concordancia que contribuye a dar mayor credibilidad a los resultados extraídos en la presente investigación. En nuestro estudio podemos ver que se confirman resultados exitosos ya remarcados por otros autores en investigaciones anteriores, tales como Lonka et al., (2004), quienes indican que el marco de ABR empodera a los estudiantes, permitiéndoles adquirir conocimientos a través de desafíos, lo que consigue marcar una notable diferencia con otros alumnos que no utilizan dicho método, y cuya puesta en marcha demuestra que el proceso de enseñanza-aprendizaje puede ser profundo, atractivo, significativo y útil.

Uno de los mayores incrementos se produce en la tasa de rendimiento (incremento del 12\%), que, atendiendo a Portuguez y Gomez (2020) se debe a que con el ABR los estudiantes profundizan y aprenden más cuando se involucran activamente para solucionar los retos. Otros autores como Kohn et al., (2020) señalan que la utilización de la metodología ABR favorece la resolución de problemas complejos, provocando mayor interés. Respecto a la evaluación, el ABR actúa también positivamente ya que implica que aquella se debe abordar con un sentido integrador (Gaskins et al., 2015), poniendo en juego actividades evaluativas que armonicen los resultados del aprendizaje con las evidencias que el alumnado aporta, lo que permite modalidades de evaluación novedosas, incluida la autoevaluación y la que incorpora actores no directamente vinculados al universo docente, como los empleadores. Por todo ello, la utilización del ABR motiva a los estudiantes a presentarse a los exámenes, tal y como indican Gallagher y Savage, (2020) en su trabajo Challenge-based 
learning in higher education: an exploratory literature review mencionan que López-Fernández et al., (2020) tras emplear esta metodología observan que el estudiante está más motivado. En lo que concierne a la tasa de éxito, el efecto de la metodología no es significativo, pero sí positivo. Estas pequeñas variaciones en los resultados, que no afectan negativamente al resultado, según los autores consultados como es el caso de Tang y Chow (2020), se deben a que no existe una única forma de aprender, ya que cada individuo interacciona de forma diferente frente al aprendizaje en función de los estilos de aprendizaje.

El acceso a enormes cantidades de información gracias a las nuevas tecnologías de la comunicación, hacen necesaria una continua adaptación de las técnicas y actividades de aprendizaje a este nuevo escenario y a sus efectos sobre el mercado laboral, combinando conocimiento académico y aplicación real (Romero-Martín et al., 2017). La metodología de ABR incluye en la ecuación enseñanza-aprendizaje elementos externos, por ejemplo, personas de otras organizaciones o empresas, que se involucran en el proceso, lo que hace que el estudiante sienta su trabajo más importante y con más sentido de responsabilidad (Agüero et al., 2019). Es, por tanto, un método escalable y aplicable a cualquier universidad con pocas necesidades de cambio y basado en una idea: hacer el aprendizaje relevante y atender a las competencias requeridas (Félix-Herrán et al., 2019).

A la luz de lo anterior, queda claro que el enfoque del ABR basa sus pilares en el desarrollo competencial, y el principio básico por el que se rige es que el alumnado debe ser capaz de construir su propio conocimiento a través de la interacción con la realidad (Ngai et al., 2020). Y ello contribuye a la mejora del proceso de incorporación de las competencias y habilidades requeridas por los empleadores, como la creatividad, competencia clave en las áreas de comunicación estudiadas y que también ha salido reforzada en otros ámbitos del conocimiento (Yang et al., 2018). Por ello, el ABR supone una innovación fundamental de cara a imbuir a los estudiantes en escenarios reales a través de proyectos y simulaciones (Gaskins et al., 2015) que ha venido para quedarse como uno de los marcos de referencia en la docencia universitaria del siglo XXI (Gallagher y Savage, 2020).

\section{CONCLUSIONES}

A la luz de los resultados de este estudio, del análisis detallado de los datos trabajados en el proceso muestral empleado y de su posterior discusión, se pueden extraer las siguientes tres conclusiones: 1) el aprendizaje basado en retos es una metodología educativa altamente positiva que genera efectos beneficiosos en el proceso de enseñanza-aprendizaje y, por ende, sobre el rendimiento de los estudiantes, cuyo impacto positivo se ha comprobado midiendo las tasas académicas (tasas de evaluación, rendimiento y éxito); 2) esta metodología contribuye a la mejora del proceso de incorporación de las competencias y habilidades requeridas por los empleadores, por lo que es necesaria su puesta en práctica para situar a los alumnos ante contextos profesionales y retos reales similares a los que tendrán que afrontar una vez se encuentren en el ámbito profesional; y 3) incluir la metodología de aprendizaje basado en retos en la rutina de aprendizaje puede aportar a cualquier universidad fórmulas basadas en la puesta en marcha de desafíos reales, donde los estudiantes pueden ver su trabajo hecho realidad.

\section{REFERENCIAS}

Agüero, M.M., López, L.A., y Pérez, J., El aprendizaje basado en retos como modelo de aprendizaje profesionalizante. Caso del programa Universidad Europea con Comunica+A, https://doi.org/10.15178/va.2019.149.124, Vivat Academia, (149), 1-25 (2019)

Bashir, M., Wee, C., Memon, N., y Guo, B., Profiling cybersecurity competition participants: Self-efficacy, decisionmaking and interests predict effectiveness of competitions as a recruitment tool, https://doi.org/10.1016/j.cose.2016.10.007, Computers \& Security, 65, 153-165 (2017)

Clausen, H.B., y Andersson, V., Problem-based learning, education and employability: a case study with master's students from Aalborg University, Denmark, https://doi.org/10.1080/15313220.2018.1522290, Journal of Teaching in Travel \& Tourism, 19(2), 126-139 (2019)

Comisión Europea. Guía de uso del ECTS 2015, Dirección General de Educación, Juventud, Deporte y Cultura (Comisión Europea) (2017)

Epstein, R.M., y Hundert, E.M., Defining and assessing professional competence, https://doi.org/10.1001/jama.287.2.226, JAMA, 287(2), 226-235 (2002)

Erice, D.B., Pardo, V.G.V., Questier, F., y Luján, D.P., La producción del conocimiento experiencial de los estudiantes en la educación superior, https://doi.org/10.19053/22160159.5216, Praxis \& Saber, 7(14), 17-39 (2016)

Félix-Herrán, L.C., Rendon-Nava, A.E., y Jalil, J.M.N., Challenge-based learning: an I-semester for experiential learning in Mechatronics Engineering, https://doi.org/10.1007/s12008-019-00569-4, International Journal on Interactive Design and Manufacturing, 13(4), 1367-1383 (2019) 
Gallagher, S.E., y Savage, T. Challenge-based learning in higher education: an exploratory literature review, https://doi.org/10.1080/13562517.2020.1863354, Teaching in Higher Education, 1-23 (2020)

Garbanzo, V.M.G., Factores asociados al rendimiento académico en estudiantes universitarios, una reflexión desde la calidad de la educación superior pública, Revista educación, ISSN: 0379-7082, 31(1), 43-63 (2007)

García, R.T.M., Factores que intervienen en el rendimiento académico universitario: Un estudio de caso, Opción, ISSN: 1012-1587, 31(6), 1041-1063 (2015)

Gaskins, W.B., Johnson, J., Maltbie, C., y Kukreti, A., Changing the learning environment in the college of engineering and applied science using challenge based learning, https://doi.org/10.3991/ijep.v5i1.4138, International Journal of Engineering Pedagogy, 5(1), 33-41 (2015)

Gómez S.C., Fernández A.E., Cerezo R., y Núñez J.C., Dificultades de aprendizaje en Educación Superior: un reto para la comunidad universitaria, https://doi.org/10.30827/publicaciones.v48i1.7328, PUBLICACIONES, 48(1), 63-75 (2018)

Kohn R.K., Lundqvist, U., Malmqvist, J., y Hagvall S.O. From CDIO to challenge-based learning experiences-expanding student learning as well as societal impact?, https://doi.org/10.1080/03043797.2018.1441265, European Journal of Engineering Education, 45(1), 22-37 (2020)

Lonka, K., Olkinuora, E., y Mäkinen, J., Aspects and prospects of measuring studying and learning in higher education, https://doi.org/10.1007/s10648-004-0002-1, Educational Psychology Review, 16, 301-323 (2004)

López-Fernández, D., Sánchez, P. S., y otros tres autores, Challenge-Based Learning in Aerospace Engineering Education: The ESA Concurrent Engineering Challenge at the Technical University of

Madrid, https://doi.org/10.1016/j.actaastro.2020.03.027, Acta Astronautica, 171, 369-377 (2020). Tomado de Gallagher y Savage (2020)

Lozano, A., García, F., Zubieta R.C., López, C.C., Competencies associated with Semestre i and its relationship to academic performance: A case study, https://doi.org/10.1108/HESWBL-07-2019-0092, Higher Education, Skills and Work-Based Learning, 10(2), 387-399 (2019)

Mandeville, D.S., Ho, T.K., y Lindy A.V., The Effect of Problem Based Learning on Undergraduate Oral Communication Competency, https://doi.org/10.19030/tlc.v14i1.9957, Journal of College Teaching \& Learning, 14(1), 1-10 (2017)

Martín, E., García, L.A., Torbay, Á., y Rodríguez, T., Estrategias de aprendizaje y rendimiento académico en estudiantes universitarios, International Journal of Psychology and Psychological Therapy, 8(3), 401-412 (2008)

Membrillo-Hernández, J., Ramírez-Cadena, M. D. J., y otros cinco autores., Challenge based learning: the case of sustainable development engineering at the Tecnologico de Monterrey, Mexico City Campus, https://doi.org/10.3991/ijep.v8i3.8007, International Conference on Interactive Collaborative Learning, 8(3), 908-914 (2018)

Mishra, P., Pandey, C.M., y otros cuatro autores, Descriptive statistics and normality tests for statistical data, https://doi.org/10.4103/aca.ACA_157_18, Annals of cardiac anaesthesia, 22(1), 67-72 (2019)

Ngai, P.B., Yoshimura, S.M., y Doi, F., Intercultural competence development via online social networking: the Japanese students' experience with internationalization in US higher education, https://doi.org/10.1080/14675986.2019.1702289, Intercultural Education, 31(2), 228-243 (2020)

O'Mahony, T. K., Vye, N. J., y otros siete autores., A comparison of lecture-based and challenge-based learning in a workplace setting: Course designs, patterns of interactivity, and learning outcomes, https://doi.org/10.1080/10508406.2011.611775, Journal of the Learning Sciences, 21(1), 182-206 (2012)

Pinto, J.A., y Soto, J.A., Challenge-based Learning: Un puente metodológico entre la educación superior y el mundo profesional, $1^{\underline{a}}$ ed., 1-332, Thomson Reuters Aranzadi, Navarra, España (2021)

Portuguez, M., y Gomez, M.G. Challenge based learning: Innovative pedagogy for sustainability through e-learning in higher education, https://doi.org/10.3390/su12104063, Sustainability, 12(10), 4063 (2020)

Romero-Martín, R., Castejón-Oliva, F.J., López-Pastor, V.M., y Fraile-Aranda, A. Formative Assessment, Communication Skills and ICT in Initial Teacher Training, https://doi.org/10.3916/C52-2017-07, Comunicar: Revista Científica de Comunicación y Educación, 25(52), 73-82 (2017)

Sistema Integrado de Información Universitaria (SIIU), Indicadores universitarios de rendimiento académico, Ministerio de Ciencia, Innovación y Universidades (2019)

Soto, M.F.M., y Amores, I.A.C., Determinantes del rendimiento académico de los estudiantes de nuevo acceso a la Universidad Complutense de Madrid, https://doi.org/10.4438/1988-592X-RE-2020-387-433, Revista de educación, (387), 213-240 (2020)

Tang, A.C., y Chow, M.C., To evaluate the effect of challenge-based learning on the approaches to learning of Chinese nursing students: A quasi-experimental study, https://doi.org/10.1016/j.nedt.2019.104293, Nurse education today, 85, 104-293 (2020)

Yang, Z., Zhou, Y., y otros 4 autores, Challenge Based Learning nurtures creative thinking: An evaluative study, https://doi.org/10.1016/j.nedt.2018.09.004, Nurse education today, 71, 40-47 (2018) 